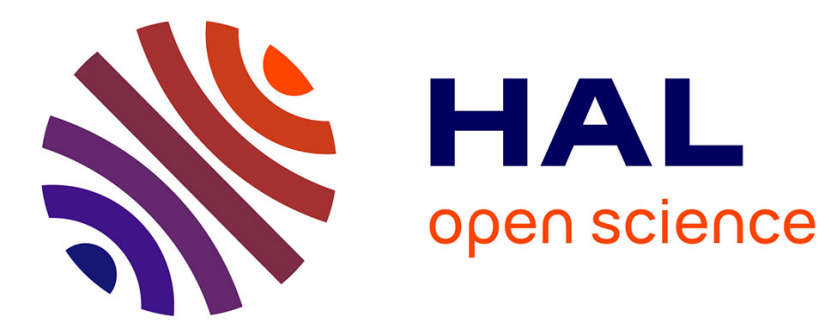

\title{
Proofs by induction in equational theories with constructors
}

\author{
Gérard Huet, J.M. Hullot
}

\section{To cite this version:}

Gérard Huet, J.M. Hullot. Proofs by induction in equational theories with constructors. [Research Report] RR-0028, INRIA. 1980. inria-00076533

\section{HAL Id: inria-00076533 \\ https://hal.inria.fr/inria-00076533}

Submitted on 24 May 2006

HAL is a multi-disciplinary open access archive for the deposit and dissemination of scientific research documents, whether they are published or not. The documents may come from teaching and research institutions in France or abroad, or from public or private research centers.
L'archive ouverte pluridisciplinaire HAL, est destinée au dépôt et à la diffusion de documents scientifiques de niveau recherche, publiés ou non, émanant des établissements d'enseignement et de recherche français ou étrangers, des laboratoires publics ou privés. 
Rapports de Recherche

$$
\mathrm{N}^{\circ} 28
$$

\section{PROOFS BY INDUCTION IN EQUATIONAL THEORIES WITH CONSTRUCTORS}

Institut National de Recherche en Informatique et en Automatique

Domaine de Voluceau Rocquencourt B.P.105: 78150 LeChesnay France Tél.9549020

\section{Gérard HUET}

Jean-Marie HULLOT 
Proofs by Induction in Equational Theories with Constructors

Gérard HUET and Jean-Marie HULLOT

Résumé

Nous montrons comment faire des démonstrations (et des réfutations) d'identités dans l'algèbre initiale d'une variété, equationnelle, par une simple extension de l'algorithme de complétion de Knuth et Bendix. Ceci nous permet de démontrer par des méthodes équationnelles des théorèmes dont la preuve nécessite d'ordinaire l'utilisation d'un principe de récurrence. Nous montrons des applications de cette méthode a des preuves de programes calculant sur des structures de données récursives, et a des preuves de somations algébriques. Ce travail étend et simplifie des résultats récents de Musser et de Goguen.

Abstract

We show how to prove (and disprove) theorems in the initial algebra of an equational variety by a simple extension of the Knuth-Bendix completion algorithm. This allows us to prove by purely equational reasoning theorems whose proof usually requires induction. We show applications of this method to proofs of programs computing over data structures, and to proofs of algebraic sumation identities. This work extends and simplifies recent results of Musser and Goguen. 


\title{
PROOFS BY INDUCTION IN EQUATIONAL THEORIES WITH CONSTRUCTORS
}

\author{
Gérard Huet and Jean-Marie Hullot
}

\section{INRIA}

\begin{abstract}
Abstrict
We show how to prove (and disprove) theorems in the initial algebra of an equational variety by a simple extension of the Knuth-Bendix completion algorithm. This allows us to prove by purely equational reasoning theorems whose proof usually requires induction. We show applications of this method to proofs of programs computing over data structures, and to proofs of algebraic summation identities. This work extends and simplifies recent results of Mussei ${ }^{15}$ and Goguen ${ }^{6}$.
\end{abstract}

\section{Introduction}

We assume familiarity with the basic notions of equational logic and term rewriting systems. See for instance ${ }^{11}$. For simplicity of notation, we assume we have only one sort; all the results of this paper carry over to many-sorted theories without difficulty.

A set of equations $\mathcal{E}$ defines a variety, that is the class of algebras which are models of the equations considered as axioms. An equation $M=N$ is said to be valid in this variety if it is true in all these models. It is well known that this is equivalent to whether $M=N$ can be derived from $\mathcal{E}$, using instantiation and replacement of equals by equals. In the cases where $\mathcal{E}$ can be compiled into a canonical term rewriting system by the Knuth-Bendix completion algorithm ${ }^{13}$, we can decide this problem by testing for identity the canonical forms of $M$ and $N$.

Equations may also be used as definitions. This is frequent in computer science: programs written in applicative programming languages, abstract interpreter definitions and algebraic data type specifications are of this nature. In this framework, one has in mind a notion of standard model defined by these equations: the initial algebra defined by the set of equations. Now

This work was partially supported by AFOSR Contract 549620 70.C-0090, by ONR Contract N00014-75-C.0816 and by a fellowship from INRLA while the autbors were visiting SRI Inter national. we have lost the nice completeness property of equational logic: an equation $M=N$ cannot in general be proved to be valid (or invalid) in the initial algebra by mere equational reasoning: some kind of induction is necessary.

However, Musser has recently shown an interesting theorem which may be roughly stated as follows: if the set of equations considered contains the axiomatization of an equality predicate, then an equation is valid in the initial algebra if and only if adding it as an axiom does not make the theory inconsistent (in the sense that true $=$ false is derivable). This permits proofs (and disproofs) of equations without explicit induction. The method was simplified by Goguen ${ }^{6}$ and Huet and Oppen ${ }^{11}$.

We show in this paper that in the case where one considers inductive definitions over free algebras, and when the Knuth-Bendix completion algorithm converges, we can make these proofs by a very simple extension of the completion algorithm, and without the need of an equality axiomatization. We show how the method applies to proofs of simple properties and optimizations of primitive recursive programs over recursively defined data structures. The inductive completion algorithm defined in the paper generates implicitly the necessary instances of structural induction. The method generalizes to commutative-associative theories, and we show an application to proofs of algebraic sum. mation identities.

\section{A Principle of Deflaition}

The key of our method consists in partitioning our function symbols between constructors and defined function symbols, and to express the necessary relationships between them via a principle of definition.

We assume given signafure $\Sigma$. Every operator $F$ in $\Sigma$ is given with its arity. The signature $\Sigma$ is partitioned as $\Sigma=C \uplus D$. We call operators in $C$ the constructors, and members of $D$ the defined operators. We assume there are at least two constructors (for instance, true and (alse). 
Let $\tau$ be the set of terms constructed from operators in $\Sigma$ and variables in a given denumerable set $v$. We use $g$ to denote the set of ground terms, i.e. containing no variables, and we assume $\mathscr{G}$ nonempty. Finally we denote by $\mathcal{G C}$ the set of ground terms formed solely from constructors.

Prineiple of DeDnition. Let $\mathcal{E}$ be a set of equations over $\Sigma,=\varepsilon$ the corresponding congruence on $\tau$. We say that $\varepsilon$ defines $D$ over $C$ if and only if for every $M$ in $g$ there exists a unique $N$ in $\mathscr{G C}$ such that $M=\varepsilon$ $N$.

It is convenient to express our principle of definition as the conjunction of two properties:

(1) For every $M$ in $G$ there exists $N$ in $\mathscr{G C}$ such that $M=\varepsilon N$.

(2) For every $M, N$ in $G C$ we have $M=\varepsilon N$ only if $M=N$.

When $\varepsilon$ satisfies (1), we shall use $\left.G C_{\varepsilon} \mid M\right]$, for $M$ in $G$, to denote any $N$ in $G C$ such that $M=\varepsilon N$. Note that (1) implies that we have a constructor signature in the sense of Goguen ${ }^{6}$. If $\mathcal{E}$ satisfies (2) as well, $\hat{y} C_{\mathcal{C}}$ is a function, and then the set $G C$ can easily be made into a $\Sigma$-algebra by associating with $F$ of arity $n$ the function $\lambda M_{1}, \ldots, M_{n} \cdot \mathscr{G} C_{\mathcal{E}}\left[F\left(M_{1}, \ldots, M_{n}\right)\right]$. Morecver:

Lemma 1. If $\mathcal{E}$ satisfies the principle of definition, the algebra $G C$ is isomorphic to the initial algebra $I(\Sigma, \mathcal{E})$.

Proof. Follows directly from the fact that the initial algebra is (isomorphic to) the quotient of $\mathcal{G}$ by $=\boldsymbol{c}$. (See for instance ${ }^{8}$ ).

\section{Sumeient Conditions for Deciding the Deflnition Prineiple}

Let us consider sufficient conditions for our principle of definition to hold. We shall from now on regard our sets of equations (when possible) as sets of (oriented) rewrite rules. We assume familiarity with the terminology of term rewriting system $\mathrm{s}^{9,11}$. In particular, we recall that a canonical term rewriting system is defined as being confluent (i.e. to have the Church-Rosser property) and nœtherian (i.e. all sequences of rewriting terminate).

Lemma 2. Let $\mathcal{E}$ be such that it defines a nœtherian term rewriting system such that every term of the form $F\left(M_{1}, M_{2}, \ldots, M_{n}\right)$, with $F$ in $D$ and $M_{1}, \ldots, M_{n}$ in $\mathcal{G C}$, is reducible. Then $\mathcal{E}$ satisfies (1).

Proof. Define $\mathscr{G} C_{\varepsilon}(M)$, for $M$ in $\mathcal{G}$, as some $\mathcal{E}$-normal form of $M$. It is easy to show by structural induction that any such normal form must be in $9 C$.
There are several ways to give effective conditions that are sufficient to entail the hypothesis of lemma 2. We shall propose here one such condition; we recommend skipping the details of the next definition on a first reading.

Defnition. We define inductively what it means for a set $S=\left\{S_{1}, \ldots, S_{p}\right\}$ of $k$-tuples of terms $S_{i}=$ $\left(S_{i}^{1}, \ldots, S_{i}^{k}\right) \quad(1 \leq i \leq p)$ to be complete for $C$. First we require every variable of $S_{i}$ to occur in only one occurrence. Then either $k=0$, and $S=\{(\mid)\}$, or else: - either the set of $k-1$-tuples $\left\{\left(S_{i}^{2}, \ldots, S_{i}^{k}\right\rangle \mid S_{i}^{1} \in V\right\}$ is complete,

- or else for every $C$ in $C$, say of arity $n$, there is at least one $S_{i}^{1}$ with leading function symbol $C$, and the union of the two sets of $n+k-1$-tuples

$\left\{\left(P_{1}, \ldots, P_{n}, S_{i}^{2}, \ldots, S_{i}^{k}\right) \mid S_{i}^{1}=C\left(P_{1}, \ldots, P_{n}\right)\right\}$ and $\left\{\left(x_{1}, \ldots, x_{n}, S_{i}^{2}, \ldots, S_{i}^{k}\right) \mid S_{i}^{1} \in V\right\}$ is complete, where the $x_{i}$ 's are new distinct variables not occurring in $S$.

Remark that this definition is well-founded, first on the number of function symbols contained in $S$, and second on $k$.

Example. With $C=\{S, 0\}$, with $S$ unary and 0 a constant, the following set is complete for $C$ :

$\{(0, S(x)\rangle,\{x, 0\rangle,\langle S(x), S(0)\rangle,\langle S(x), S(S(y))\}\}$.

Lemma 3. Let $S=\left\{S_{1}, \ldots, S_{p}\right\}$ be a set of $k$-tuples of terms complete for $C$. For every $k$-tuple of ground terms in $\mathscr{G C}:\left\langle M_{1}, \ldots, M_{k}\right\rangle$ there exist $n$, with $1 \leq$ $n \leq p$, and a substitution 0 , such that for every $l$, $1 \leq \ell \leq k$, we have $M_{\ell}=\sigma\left(S_{n}^{\ell}\right)$

Proof. Easy induction on the definition of complete. Pro

This lemma permits us to state a sufficient condition for property (1), which we shall use in practice:

Lemma 4. Let $\mathcal{E}$ be a set of equations defining a nœtherian term rewriting system such that, for every $F$ in $D$, there is in $\mathcal{E}$ a set of rewrite rules whose lefthand sides are of the form $F\left(S_{i}^{1}, \ldots, S_{i}^{k}\right),(1 \leq i \leq p)$, and the set $\left\{S_{1}, \ldots, S_{p}\right\}$ is complete for $C$. Then $\mathcal{E}$ has property (1).

Proof. It is easy to show, using lemma 3, that the assumption of the lemma implies that of lemma 2.

Remark that if $\mathcal{E}$ is finite and known to be nctherian, then the hypothesis of the lemma is a decidable condition. Actually, when giving the definition of $F$ in $D$ by cases on arguments constructed over $C$, one naturally gets complete sets of arguments.

Finally we state a trivial sufficient condition for property (2). 
Lemma 5. Let $\mathcal{E}$ be a set of equations defining a canonical term rewriting system such that every lefthand side is of the form $F\left(M_{1}, \ldots, M_{n}\right)$ with $F$ in $D$. Then $\mathcal{E}$ has property (2).

Proof. Since $\varepsilon$ is canonical, we have $M=\varepsilon N$ if and only if $M \downarrow=N \downarrow$, where $M \downarrow$ denotes the canonical form of $M$ obtained by an arbitrary terminating sequence of rewritings by rules in $\mathcal{E}$. If all left-hand sides of equations in $\varepsilon$ have their leading function symbol in $D$, we have $M \downarrow=M$ for every $M$ in $g C$.

Putting together the two preceding lemmas gives a useful sufficient criterion for the principle of definition to hold. For instance, any set of primitive recursive definitions satisfies the hypotheses of lemmas 4 and 5 .

Remark that if $\mathcal{E}$ obeys the hypothesis of lemma 5 , then the converse of lemma 2 holds: $\mathcal{E}$ satisfies the definition principle if and only if $\mathcal{G C}$ is the set of $\mathcal{E}$-normal forms, and then $\mathcal{G} C_{\mathcal{C}}|M|$ is the canonical form of $M$ defined by $\mathcal{E}$. However, the converse of lemma 4 may not hold, since property (1) may be the consequence of axioms in $\varepsilon$ whose left hand sides contain multiple occurrences of a variable. We shall return to this problem in section 5 .

\section{Structural Ioduction and the Principle of Defnition}

In this section, we shall show how our principle of definition permits us to prove and disprove properties of the standard model $I(\Sigma, \varepsilon)$. The next lemma shows that the principle of definition is preserved by extension if and only if this extension is valid in the standard model.

Lemma 6. Let $\mathcal{E}$ satisfy (1) above. Let $\mathcal{E}^{\prime}$ be any set of $\boldsymbol{\Sigma}$-equations such that $=\epsilon$ is contained in $=\boldsymbol{C}^{\prime}$. Then $\mathcal{E}^{\prime}$ satisfies (2) if and only if:

a) $\varepsilon$ satisfies (2), and

b) every equation of $\mathcal{E}^{\prime}$ holds in $I(\Sigma, \varepsilon)$.

Proof. Obviously, $\varepsilon^{\prime}$ satisfies (1), and it satisñes (2) only if $\mathcal{E}$ does too.

$\Rightarrow$ Assume that $\varepsilon^{\prime}$ satisfies (2) and that $M=N$ in $\mathcal{E}^{\prime}$ does not hold in $I(\Sigma, \mathcal{E})$. This means that for some ground substitution $\sigma$ we have $\sigma(M) \neq \varepsilon \sigma(N)$. In particular we get $G C_{\varepsilon}[\sigma(M)] \neq \mathcal{G} C_{\varepsilon}[\sigma(N)]$, although $G C_{\varepsilon}[\sigma(M)]=\varepsilon^{\prime}, C_{C}[\sigma(N)]$, a contradiction with (2) for $\mathcal{E}^{\prime}$.

$\Leftarrow$ If every equation of $\mathcal{E}^{\prime}$ holds in $I(\Sigma, \mathcal{E})$, then for every $M, N$ in $\mathcal{G}$ we have $M={ }^{\prime} N$ if and only if $M=\varepsilon N$, and (2) for $\varepsilon^{\prime}$ follows from (2) for $\varepsilon$.

The next three lemmas give technical properties of equality in the standard model that are essential to the proof of our completion algorithm.
Lemma 7. Let $M=C\left(M_{1}, \ldots, M_{n}\right), N=C\left(N_{1}, \ldots\right.$, $\left.N_{n}\right)$, with $C$ in $C$. Let $\mathcal{E}$ be a set of equations satisfying the principle of definition such that $M=c N$. Then $M_{i}=N_{i}$ holds in $I(\Sigma, \mathcal{E})$ for every $i, 1 \leq i \leq n$.

Proof. Let $\sigma$ be an arbitrary ground substitution, and assume $M=c N$. We have

$$
\begin{aligned}
\sigma(M) & =C\left(\sigma\left(M_{1}\right), \ldots, \sigma\left(M_{n}\right)\right) \\
& =\varepsilon C\left(\sigma\left(N_{1}\right), \ldots, \sigma\left(N_{n}\right)\right)=\sigma(N)
\end{aligned}
$$

and by (1) we get

$$
\begin{aligned}
& C\left(\mathscr{G} C_{\varepsilon}\left[\sigma\left(M_{1}\right)\right], \ldots, g C_{\varepsilon}\left[\sigma\left(M_{n}\right)\right]\right) \\
& =\varepsilon_{\varepsilon} C\left(\mathscr{G} C_{c}\left[\sigma\left(N_{1}\right)\right], \ldots, g C_{\varepsilon}\left[\sigma\left(N_{n}\right)\right]\right)
\end{aligned}
$$

which implies by (2) $\mathcal{G} C_{\varepsilon}\left[\sigma\left(M_{i}\right)\right]=\mathscr{G} C_{\varepsilon}\left[\sigma\left(N_{i}\right)\right]$ for every $i, 1 \leq i \leq n$, and thus $\sigma\left(M_{i}\right)=c \sigma\left(N_{i}\right)$. Since this holds for every ground $\sigma$, we get that $M_{i}=N_{i}$ holds in $I(\Sigma, \mathcal{E})$.

Corollary. With $M$ and $N$ as above, let $\varepsilon$ containing $M=N$ and satisfying (1). Consider $\mathcal{E}^{\prime}=\mathcal{E}-\{M=$ $N\} \cup\left\{M_{i}=N_{i} \mid 1 \leq i \leq n\right\}$. Obviously $=\varepsilon$ is contained in $=\varepsilon$. Now either $\mathcal{E}$ satisfies (2), in which case $I\left(\Sigma, \mathcal{E}^{\prime}\right)=I(\Sigma, \mathcal{E})$ by lemma 7 , and $\mathcal{E}^{\prime}$ satisfies (2) by lemma 6 , or else $\mathcal{E}^{\prime}$ does not satisfy (2).

Lemma 8. Let $M=C\left(M_{1}, \ldots, M_{n}\right), N=D\left(N_{1}, \ldots\right.$, $\left.N_{p}\right)$, with $C$ and $D$ two distinct constructors. Let $\mathcal{E}$ be a set of equations satisfying (1) and such that $M=\varepsilon$ $N$. Then $\mathcal{E}$ does not satisfy (2).

Proof. Let $\sigma$ be any substitution substituting ground terms for every variable occurring in $M$ or $N$. From $M=\varepsilon N$ we get

$$
\begin{aligned}
\sigma(M) & =C\left(\sigma\left(M_{1}\right), \ldots, \sigma\left(M_{n}\right)\right) \\
& =\varepsilon D\left(\sigma\left(N_{1}\right), \ldots, \sigma\left(N_{p}\right)\right)=\sigma(N)
\end{aligned}
$$

and therefore by (1)

$$
\begin{aligned}
& C\left(\mathcal{G C}_{c}\left[\sigma\left(M_{1}\right)\right\}_{1} \ldots, g C_{c}\left[\sigma\left(M_{n}\right)\right]\right) \\
& ={ }_{\varepsilon} D\left(\mathcal{G} C_{\varepsilon}\left[\sigma\left(N_{1}\right)\right], \ldots, g C_{\varepsilon}\left[\sigma\left(N_{p}\right)\right]\right)
\end{aligned}
$$

a contradiction with (2).

Lemma 9. Let $M=C\left(M_{1}, \ldots, M_{n}\right)$, with $C$ in $C$, and let $N$ be a variable. Let $\mathcal{E}$ be a set of equations satisfying (1) and such that $M=\varepsilon N$. Then $\varepsilon$ does not satisfy (2).

Proof. Let $\sigma$ be any substitution that replaces $N$ by a term whose leading function symbol is a constructor distinct from $C$ (Remember that we assume the existence of at least two constructors.) We have $\sigma(M)=\varepsilon$ $\sigma(N)$, and the result follows from the preceding lemma $\theta$

We are now ready to present our extension of the Knuth-Bendix completion algorithm.

\section{The Inductive Completion Algorithm}

Let $\mathcal{E}$ satisfying the principle of definition, $\mathcal{C}^{\prime}$ any set of $\Sigma$-equations. Run the Knuth-Bendix completion 
algorithm on $\mathcal{E} \cup \mathcal{E}^{\prime}$, with the following modifications. We assume given a well-founded partial ordering on terms $\succ$, compatible with the term structure and stable by substitution, with which we prove the termination of the successive sets of rewrite rules. We assume familiarity with the Knuth-Bendix completion algorithm, as presented for instance in Huet ${ }^{10}$. The only modification occurs in the step in which a pair of terms, coming from either a simplifed critical pair or a reduced rewrite rule, is considered for orientation before being added as a new rewrite rule. This step should be modified as follows, assuming that $(M, N)$ is the current candidate rewrite rule, with $M \neq N$.

- If $M=C\left(M_{1}, \ldots, M_{n}\right)$ with $C$ in $C$, then:

- If $N=C\left(N_{1}, \ldots, N_{n}\right)$ then replace the pair by the $n$ pairs $\left\langle M_{i}, N_{i}\right\rangle$

- If $N=D\left(N_{1}, \ldots, N_{p}\right)$, with $D$ in $C, D \neq C$, or $N=x$ stop "disproof"

- Otherwise:

- If $N \succ M$, introduce new rule $N \rightarrow M$

- Otherwice stop "failure"

- Otherwise:

- If $N=C\left(N_{1}, \ldots, N_{n}\right)$ with $C$ in $C$ do symetrically as above

- Otherwise:

- If $M \succ N$, introduce new rule $M \rightarrow N$

- If $N \succ M$, introduce new rule $N \rightarrow M$

- Otherwise stop "failure".

The new inductive completion algorithm may:

- stop with success, i.e. we get a finite canonical term rewriting system.

- stop with disproof.

- stop with failure, i.e. either the ordering $>$ used was inadequate to prove the termination of the set of current rewrite rules, or this set is nonterminating, and the method is therefore not applicable.

- run forever, generating an infinite set of rewrite rules.

Theorem. If the algorithm stops with success, every equation in $\mathcal{E}^{\prime}$ holds in $I(\Sigma, \mathcal{E})$. Furthermore, the resulting canonical term rewriting system satisfies the principle of definition. If the algorithm stops with disproof, some equation in $\mathcal{E}^{\prime}$ does not hold in $I(\Sigma, \mathcal{E})$. Conversely, if some equation in $\mathcal{E}^{\prime}$ does not hold in $I(\Sigma, \varepsilon)$, the algorithm stops with either disproof or failure.

Proof. From the lemmas above and the properties of the Knuth-Bendix algorithm, as proved in Buet ${ }^{10}$.
Note that lemma 5 forbids us to introduce a lefthand side whose main function symbol is a constructor. This is necessary, as shown by the following example. Let $C=\{A, B\}, D=\{C\}$, all symbols nullary. Let $\varepsilon=\{C=A\}$, and $\varepsilon^{\prime}=\{C=B\}$. With $A>C$ and $B \succ C$, the usual completion algorithm would converge with the canonical set $\{A \rightarrow C, B \rightarrow C\}$. The algorithm above would oblige us to use an ordering such that $C>A$ and $C>B$, would construct for $E U$ $\varepsilon^{\prime}$ the term rewriting system $\{C \rightarrow A, C \rightarrow B\}$, and the critical pair $(A, B\rangle$ would (correctly) force stopping with disproof.

The theorem above was inspired by the work of Musser ${ }^{15}$, and its extensions in the taut presentations of Goguen ${ }^{6}$ and the s-separable theories of Huet and Oppen ${ }^{11}$. However, note that here no special equality axioms are required.

\section{General Organization of Inductive Proofs}

Assume we are working in the initial theory $I(\Sigma, \mathcal{E})$, with $\Sigma=C \biguplus D$. That is, we are interested in studying properties of the objects freely constructed from members of $C$, and to this end we have axiomatized the operators of $D$ using the equations in $\mathcal{E}$ as recursive definitions.

We check that every left-band side of $\mathcal{E}$ is of the form $F\left(M_{1}, \ldots, M_{n}\right)$ with $F \in D$, that $\mathcal{E}$ is confluent, ncetherian, and verifies the hypothesis of lemma 4. These checks usually go together: if $\mathcal{E}$ is a set of primitive recursive-like definitions, it can be proved notberian by a simple lexicographic ordering argument, every defined function symbol has trivially a complete set of arguments, and the set is confluent because there are no critical pairs.

Now let $\mathcal{E}^{\prime}$ be a set of equations which we conjecture about the inductive theory above. We run the inductive completion algorithm above, initializing the set of rewrite rules to $\mathcal{E}$ and the set of equations to $\mathcal{E}^{\prime}$. If the algorithm stops with failure, nothing interesting may be said. If it stops with disproof, some equation from $\varepsilon^{\prime}$ does not hold in the theory. If it stops with success, generating a canonical set $\varepsilon_{1}$, all of the equations from $\mathcal{E}^{\prime}$ are true in $I(\Sigma, \mathcal{E})=I\left(\Sigma, \mathcal{E}_{1}\right)$, and furthermore $\mathcal{E}_{1}$ satisfies the definition principle. We may therefore iterate the process, trying a new set of conjectures $\mathcal{E}_{1}^{\prime}$, while profiting of the previously proved conjectures as lemmas.

Let us now consider the situation when we want to enrich our theory with new function symbols. First of all, we remark that it would be unsound to add new constructors, since a theory complete for $C$ might not be complete for some extended $C^{\prime}$. Furthermore 
we may have proved some theorem valid in $I(\Sigma, \mathcal{E})$ which is not valid in the extended theory $I\left(\Sigma^{\prime}, \varepsilon^{\prime}\right)$. For instance, with $C=\{A, B\}$ and $\mathcal{E}=\{F(A)=$ $A, F(B)=B$ \} we may prove $F(x)=x$, but this formula is not valid any more if we extend our theory with constructor $C$ and definition $F(C)=A$. We shall therefore assume that our set of constructors $C$ is constant, and that we only permit to enrich our signature by adding new defined function symbols. We are sure this way that our extensions are mouotonous, in the sense that any theorem proved in a theory is still true in an extended theory, even if we do not keep it around as a lemma.

Assume therefore that we are currently dealing with a set of equations $\mathcal{E}$ that is knows to satisfy the definition principle, and that we are adding a new function symbol $F$ and some new definitions $\mathcal{E}^{\prime}$. How do we know that $\mathcal{E} \cup \mathcal{E}^{\prime}$ satisines the definition principle? Our problem is that $\varepsilon$ itself may not satisfy the hypothesis of lemma 4 , because $\varepsilon$ may bo obtained after some steps of completion that may have desticyed the completeness property. For instauce, consider $C=$ $\{$ true, false $\}, D=\{V\}, \mathcal{E}=\{$ true $\vee x=$ true,$x \vee$ true $=$ true, false $\vee$ false $=$ false $\}$. If we attempt to prove the theorem $x \vee x=x$ using the cumfitstion algorithm, we shall stop with succese, generaiing the canonical sei $\varepsilon^{\prime}=$ \{truc $\vee x=$ irue, $z \vee$ true $=$ true, $x \vee x=x$. This set is known to satis!y the definition principle, but lemma 4 does not apply to it any more, and therefore cannct be used to show that some extension of it satisfies the definition principle fo: an extended signature. However, the following slight generalization of lemmas 4 and 5 will be encugh to teli us how to enrich canonical theories wbile preserving the definition principle.

Assume that $\varepsilon$ obeys the hypothesis of lemma 5 and is known to have property (1) relatively to a given signature $\Sigma=C \uplus D$. Now assume we want to errich our theory by one more defined symbol $F$, i.e. we now consider signature $\Sigma^{\prime}=C \uplus(D \cup\{F\})$. Consider any set $\mathcal{E}^{\prime}$ obtained from $\mathcal{E}$ by adding a complete definition for $F$, i.e. a set of equations with left-hanci sides of the form $F\left(S_{i}^{1}, \ldots, S_{i}^{n}\right)$, the argument tupies $S_{i}$ 's forming a complete set. If $\mathcal{E}^{\prime}$ is canonical, it satisfes the principle of definition for the extended signature. This way we know how to test the validity of our definition principle in an incremental way. Actually, remark that the process of enriching a theory, once the completeness property has been checked, is exactly the same as proving lemmas: it just consists in running the completion algorithm. This is probably the most surprising feature of our theorem-prover: we treat new axioms and conjectures to be proven is exactly the same manner.

In practice it will be useful to deal with sorted theories. Over sorted theories, we shall be able to introduce new constructors, provided we introduce at a time all constructors of a given sort, and that none of the symbols considered so far bad arguments of the new sort. For instance, we may consider introducing sort bool ean with constructors true and false, define certain boolean connectives and prove properties about them, then introduce sort integer with constructors 0 and $S$, prove arithmetic properties, then introduce 1 ist-of-integers with constructors $\mathrm{Null}$ and $L i s t$, etc...

The $\Lambda$ ppendix presents examples of proofs and disproofs using the method above. All our examples satisfy the hypotheses of lemmas 4 and 5 above, as the reader may readily check. However, in the current implementation these conditions are not checked automatically. We plan to implement fully the method above, using for the termination tests recent criteria developed by Plaisted ${ }^{17}$, Dershowitz ${ }^{4}$, and Kamin \& Léry ${ }^{12}$.

\section{Fxtension to Commutative-Associative Operators}

The theory above can be extended without difficulty to the generalization of the Knuth-Bendix completion algorithm to the case where certain function symbols are assumed to be commutative and associative 14,16. These operators must be placed in $D$. For these operators, the notion of a complete set of tuples of arguments extends naturally to the notion of a complete set of multisets of arguments.

In the Appendix we apply this technique to proofs of simple arithmetic identities. In particular, we show that the sum of the first $n$ odd integers is $n^{2}$, and that the sum of the first $n$ integers is $\frac{n(n+1)}{2}$.

It appears possible too to introduce commutativeassociative contructors. This would allow proofs of recursive programs over data types sucb as multisets. However, lemma 7 must be changed accordingly; that is, an equation $C\left(M_{1}, \ldots, M_{n}\right)=C\left(N_{1}, \ldots, N_{p}\right)$, with $C \in C$ and $C$ commutative-associative, does not necessarily imply pairwise equality of the arguments $M_{i}$ and $N_{i}$. It rather implies one out of the possibly several solutions to the corresponding multiset equation. This would complicate the general organization of the method, since the proofs would have to split according to the varicus cases. W'e bave not yet implemented this mechanism in our proof syslem. 


\section{Conclusion}

We have piesented in this paper a very simple metiod to construct proofs by structural induction. The inethod is based on a straightforward modification of the Knuth-Bendix completion algorithm, and does not require an equality axiomatization. The method is simple to implement, and when it applies the proofs obtained are surprisingly short. For instance, given the two recursive definitions of the concatenation of lists, we can prove the associativity of concatenation by simply checling that this set of three equations, crisidered as rewrite rules, forms a canonical set.

Experimental evidence with an implementation of unr method suggest.s that it is powerful enough to apply to the usual proofs of correctness of algebraic data types impleneatation, and proofs of simple primitive recustive progians computing on data types such as integers, lists ard trees. We know how to handle simple fragkents of arithmetic, and thus generate automaticaily prosfs of siandard sumpation identities.

ine metiod has many limitations however. The reciaruent on tinile termination, while natural for : acisive defuitions (or recursive programs, provided we iectrict ours aives lo programs that always lerminate may be impossibie to enfoice for complex combinatiuns oí lemuas. Wie do not know bow to hadole permutaive equations, except, for conmutative-associative laws. Even when we know how to give an orientation to ail the generated equations so that finite termination hoids, the completion process may loop. It may however be possible to recognize easy patterns of such loopings, and avoid these by appropriate "metarules", such as the gereralization technique of Boyer and Mocie. Finaliy, most nontrivial program proofs involve a fair amount of propositional calculus (such as cases enalysis). Such reasoning is better dealt with as a separate top-level proof system rather than by equationa! encoding

\section{Apperdis}

The following is the image of a computer session rus of SPI's KL10 using the VLISP interpreter developed at Université de Vincennes. User input appears after question marks. When in doubt, the system asks the user the orientation of equation $M=N$ with the prompt COMMAND? to which one answers y (resp. n) to get the rewrite : ule $M \rightarrow N$ (resp. $N \rightarrow M$ ). Comments are inclosed between semi-colons.

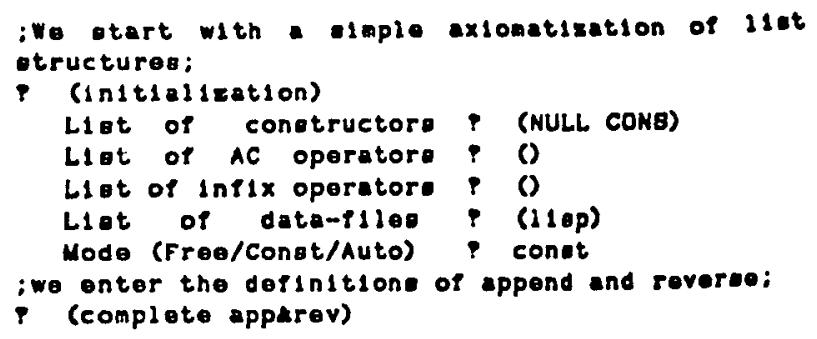

Given eet of equatione: APPaREV

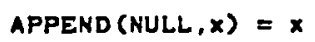

$\operatorname{REV}(\operatorname{CQNB}(x, y))=\operatorname{APPEND}(\operatorname{REV}(y), \operatorname{CONB}(x, N U L L))$ aIven

Command $y$

R4 : $\operatorname{REV}(\operatorname{CONB}(x, y)) \rightarrow \operatorname{APPEND}(\operatorname{REV}(y), \operatorname{CONB}(x, \operatorname{NULL}))$

Complete Bet: APPAREY

Unifleation time: 113mo

Rewriting time: 280me

iwe now prove rev $(\operatorname{rev}(x))=x$;

P (prove rov.rev)

alven pet of equations: REV.REV

$\operatorname{REV}(\operatorname{REV}(x))=x$

R5 : REV $(\operatorname{REV}(x)) \rightarrow x \quad$ Givon

R6 : REV (APPEND $(R E V(x), \operatorname{CONB}(y, \operatorname{NULL}))) \rightarrow \operatorname{CONB}(y, x)$

From Rs and $R$ e

RT : $\operatorname{REV}(A P P E N D(x, \operatorname{CONB}(y, N U L L))) \rightarrow \operatorname{CONB}(y, \operatorname{REV}(x))$

From $R 6$ and $R$

R6 deleted

Rewrite rules: RT RS for left part

Complete Bet: ARV.REV

Unitication time: 110me

Rewriting time: 1003me

? (anow trev.rev) 
- REV. REV

APPEND (NULL, $x) \rightarrow x$

$\operatorname{APPEND}(\operatorname{CON} B(x, y), x) \rightarrow \operatorname{CONB}(x, \operatorname{APPEND}(y, z))$

REV (NULL) $\rightarrow$ NULL

$\operatorname{REV}(\operatorname{CONB}(x, y)) \rightarrow \operatorname{APPEND}(\operatorname{REV}(y) \cdot \operatorname{CONB}(x, \operatorname{NULL}))$

REV (REV $(x)) \rightarrow x$

REV $(A P P E N D(x, \operatorname{CONB}(y, N U L L))) \rightarrow \operatorname{CONB}(y, \operatorname{REV}(x))$

: Let ue now conelder a new function brev.

in peeude-liap notation. we would program:

brev $(x)=1 f$ nuld $(x)$ then nil

elelf null $(\operatorname{cdr}(x))$ then liot $(x)$

el ae cone(car (brev $(\operatorname{cdr}(x)))$.

brov(cons $\operatorname{car}(x), \operatorname{brev}(\operatorname{cdr}(\operatorname{brov}(\operatorname{cdr}(x)))))))$.

yo ehall hero define brev with the holp of euxldiery

runcticne bravd and brev2, ouch that

brev $1(x, y)=\operatorname{car}(\operatorname{brov}(\operatorname{con} \theta(x, y)))$ and

breva $(x, y)=\operatorname{cdr}(\operatorname{brev}(\operatorname{cone}(x, y)))$.

Note that our "programo" are closer to Buretall.

HOPE than to LIBP:

- (completa rey.rer orev)

Oivan ayt of Gquatione: BREV

EREV (NULS) = NUL:

$\operatorname{BREV}(\operatorname{CONS}(x, y))=\operatorname{CONB}(\operatorname{BREV} 1(x, y), \operatorname{BREV} z(x, y))$

EREA $1(x, N$ NL.i.) $=x$

BREVI $(x, \operatorname{coNG}(y, z)=\operatorname{BREV} 1(y, z)$

BREV? $(X, N \cup 1 . L): N U ! L$

$\operatorname{BREV} z(x, \operatorname{CON} 2(y, z))=\operatorname{BREV}(\operatorname{CON} 8(x, \operatorname{BREV}(\operatorname{BREV} 2(y, x))))$

RT : BREV (XULL) $\rightarrow$ NULL

Gren

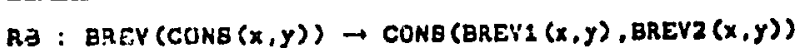

Given

R9: BREVI $(x$, NULL $) \rightarrow x$

atvan

$\operatorname{BREV} 1(x, \operatorname{CONB}(y, 8))=\operatorname{BREV} 1(y, x)$

Given

Command $P y$

R10: $\operatorname{BREV} i(x, \operatorname{CONB}(y, z)) \rightarrow \operatorname{BREV} 1(y, z)$

R11 : BREV2 (X, NULL) $\rightarrow$ NULL

olvon

R12 : BREV2 $(x, \operatorname{CONB}(y, z)) \rightarrow \operatorname{CONS}(B R E V 1(x, \operatorname{BREV}($ BREV $2(y, z))), \operatorname{BREV} 2(x, \operatorname{BREV}(\operatorname{BREV} 2(y, z))))$ Given

Complete Bet: - BREV

Unificetion time: S5Tme

Rewrlting time: 695mo

P (ohow obrov)
- brev

APPEND (NULL, $x$ ) $\rightarrow x$

$\operatorname{APPEND}(\operatorname{CONB}(x, y), z) \rightarrow \operatorname{CONB}(x, \operatorname{APPEND}(y, z))$

REY (NULL) $\rightarrow$ NULL

$\operatorname{REV}(\operatorname{CONB}(x, y)) \rightarrow \operatorname{APPEND}(\operatorname{REV}(y), \operatorname{CONB}(x, N U L L))$

REV (REV $(x)) \rightarrow x$

REV $(A P P E N D(x, \operatorname{CONB}(y, N U L L))) \rightarrow \operatorname{CONB}(y, \operatorname{REV}(x))$

BREV (NULL) $\rightarrow$ NULL

$\operatorname{BREV}(\operatorname{CONB}(x, y)) \rightarrow \operatorname{CONB}(\operatorname{BREV} 2(x, y), \operatorname{BREV} z(x, y))$

BREV1 $(x, N U L L) \rightarrow x$

BREV $1(x, \operatorname{CONB}(y, z)) \rightarrow \operatorname{BREV} 1(y, z)$

BREVZ $(x$, NULL $) \rightarrow$ NULL

$\operatorname{BREV} 2(x, \operatorname{CONB}(y, z)) \rightarrow \operatorname{CONB}(\operatorname{BREV} 1(x, \operatorname{BREV}(B R E V Z(y$. F)), BREVZ $(x, \operatorname{BREV}(\operatorname{BREV} 2(y, z))))$

ibrer la ectually etill another reveree sunction. ae we now ohow:

p (prove rev.brov)

olven oet of equatione: REV.BREV

BREV $(\dot{x})$ =REY $(x)$

$\operatorname{BREV}(x)=\operatorname{REV}(x)$

aron

Command $y$

R13 : BREV $(x) \rightarrow \operatorname{REV}(x)$

RT deleted

Rewrite rulee: R13 R3 for left part

Re roplaced by :

APPEND (REV $(x), \operatorname{CONB}(y, N U L L))=\operatorname{CONB}(\operatorname{BREV} 1(y, x)$,

Rewrito rulog: R13 R4 for left pert BREV $2(y, x))$

R12 replaced by:

$\operatorname{BREV} 2(x, \operatorname{CONB}(y, z))=\operatorname{CONB}(\operatorname{BREV} 1(x, \operatorname{REV}(\operatorname{BREV} z(y$, z)) , BREV $2(x, \operatorname{REV}(\operatorname{BAEV} 2(y, z))))$

Rewrite rulee: R13 for right part

R14: $\operatorname{BREV} 2(x, \operatorname{CONB}(y, z)) \rightarrow \operatorname{CONB}$ (BREVI $(x, \operatorname{REV}$ (BREV2 (y s)), $\operatorname{BaEV} 2(x, \operatorname{REV}(\operatorname{BREV} 2(y, z))))$ From RL2

R1D : APPEND (REV $(x), \operatorname{CONB}(y, N U L L)) \rightarrow \operatorname{CONB}(B R E Y \perp(Y$, $x), \operatorname{BREV} 2(y, x))$ From Ra

R4 replaced by:

$\operatorname{REV}(\operatorname{CON} B(x, y))=\operatorname{CONB}(\operatorname{BREV} 1(x, y), \operatorname{BREV} z(x, y))$

Rewrlte rulen: RIS for right part

R16 : REV $(\operatorname{CONB}(x, y)) \rightarrow \operatorname{CONB}(\operatorname{BREV} 2(x, y), \operatorname{BREV} 2(x, y))$ From RS

R17: BREV1 (BREV1 $(x, y), \operatorname{BREV} 2(x, y)) \rightarrow x$

From 216 and $\mathrm{Rs}$

R18: BREVZ (BREV1 $(x, y)$, BREV $2(x, y)) \rightarrow y$

Fron R26 and RS 
R18 : APPEND $(x, \operatorname{CONB}(y, N U L L)) \rightarrow \operatorname{CONB}(B R E V I(y, \operatorname{REV}(x))$. $\operatorname{BREV} 2(y, \operatorname{REV}(x)))$

From R1S and $R$ S

R6 deleted

Rewrito rulog: RIQ RI6 RI7 RIB for loft part

R15 deleted

Rewrite ruleo: R19 R5 R5 for loft part

Complete Bot: HEV. BREV

Uniflcation time: $1684 \mathrm{mo}$

Rewriting timo: 6433ng

- REV. BREV

APPEND (NULL, $x$ ) $\rightarrow x$

$\operatorname{APPEND}(\operatorname{CON} B(x, y), z) \rightarrow \operatorname{CONB}(x, \operatorname{APPEND}(y, z))$

REV (NULL) $\rightarrow$ NULL

REV (REV $(x)) \rightarrow x$

BREVI $(x, N U L L) \rightarrow x$

BREVI $(x, \operatorname{CONB}(y, z)) \rightarrow \operatorname{BREV} 1(y, z)$

BREV2 $(x$, NULL $) \rightarrow$ NULL

BREV $(x) \rightarrow \operatorname{REV}(x)$

BREV $2(x, \operatorname{CONB}(y, z)) \rightarrow \operatorname{CONB}(\operatorname{BREV} 1(x, \operatorname{REV}(\operatorname{BREV} 2(y, z)))$ BREV $2(x, \operatorname{REV}(\operatorname{BREV} 2(y, z))))$

$\operatorname{REV}(\operatorname{CONB}(x, y)) \rightarrow \operatorname{CONB}(\operatorname{BREV} 1(x, y), \operatorname{BREV} 2(x, y))$

BREV 1 (BREV $1(x, y)$, BREV $2(x, y)) \rightarrow x$

BREY2 (BREV1 $(x, y), \operatorname{BREV} 2(x, y)) \rightarrow y$

$A P P E N D(x, \operatorname{CONB}(y, N U L L)) \rightarrow \operatorname{CONB}(\operatorname{BREV} 1(y, \operatorname{REV}(x))$. BREV2 $(y, \operatorname{REV}(x)))$

inote the uee of our induction rule in the proos obove, In generating R1T and R18.

Lot ue now play with "dlotributive cone-

P (complete dcone)

olven oet of equetione: DCONB

$\operatorname{DCONB}(x, N U L L)=N U L L$

$\operatorname{DCONB}(x, \operatorname{CONB}(y, z))=\operatorname{CONB}(\operatorname{CONB}(x, y), \operatorname{DCONB}(x, z))$

$R 1: \operatorname{DCONB}(x, N U L L) \rightarrow$ NULL Given

$R 2: \operatorname{DCONB}(x, \operatorname{CONB}(y, z)) \rightarrow \operatorname{CONB}(\operatorname{CONB}(x, y), \operatorname{DCONB}(x, z))$

olven

Complete Bot: - DCONB

Undication time: 60me

Rewriting time: $28 \mathrm{me}$

; we now enter the function 1 terate, and prove

lemme relating 1 terate and dcone;

p (prove 1terato)

Given oot of equationa: ITERATE

ITERATE (NULL, $x)=N U L L$

ITERATE $(\operatorname{CONB}(x, y), z)=\operatorname{CONB}(z, \operatorname{ITERATE}(y, z))$

$\operatorname{DCONB}(x, I T E R A T E(y, z))=\operatorname{ITERATE}(y, \operatorname{CONB}(x, z))$

R3 : ITERATE (NULL, $x) \rightarrow$ NULL

G1 yen
R4 : ITERATE $(\operatorname{CONB}(x, y), z) \rightarrow \operatorname{CONB}(s, \operatorname{ITERATE}(y, z))$

aren

$\operatorname{DCONB}(x, \operatorname{ITERATE}(y, z))=\operatorname{ITERATE}(y, \operatorname{CONB}(x, z))$ GIVON Command $? y$

RD: $\operatorname{DCONB}(x, \operatorname{ITERATE}(y, z)) \rightarrow \operatorname{ITERATE}(y, \operatorname{CONB}(x, z))$

Complete Bet: iterate

Unification time: $155 \mathrm{mo}$

Rewriting time: 395me

iwe now enter the definftion of va. Iunction that repeate a vector a a matrix;

(complete 1 terate $\mathrm{vm}$ )

Given eet of equations: vu

VM (NULL) $=$ NULL

$\operatorname{VM}(\operatorname{CONB}(x, y))=\operatorname{CONB}(\operatorname{CoNs}(x, y), \operatorname{DCONB}(x, V M(y)))$

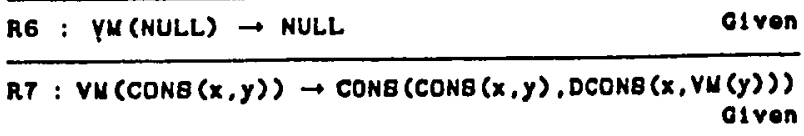

Complete Bot: vi

Unification time: $99 \mathrm{mo}$

Rewriting time: 73mo

- (ohow *vm)

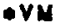

$\operatorname{DCONB}(x$, NULL $) \rightarrow$ NULL

$\operatorname{DCONB}(x, \operatorname{CONB}(y, z)) \rightarrow \operatorname{CON} \theta(\operatorname{CON} \theta(x, y), \operatorname{DCONB}(x, z))$

ITERATE $(N U L L, x) \rightarrow$ NULL

ITERATE $(\operatorname{CONB}(x, y), z) \rightarrow \operatorname{CONB}(z, \operatorname{ITERATE}(y, z))$

$\operatorname{DCONB}(x, \operatorname{ITERATE}(y, z)) \rightarrow \operatorname{ITERATE}(y, \operatorname{CONB}(x, z))$

VM (NULL) $\rightarrow$ NULL

$\operatorname{VM}(\operatorname{CONB}(x, y)) \rightarrow \operatorname{CONB}(\operatorname{CoNB}(x, y), \operatorname{DCONB}(x, V M(y)))$

;we now exprese vm in terne of iterate

- (prove vm.1terate)

alven set of equationa: VM.ITERATE

$\operatorname{VM}(x)=\operatorname{ITERATE}(x, x)$

$V N(x)=\operatorname{ITERATE}(x, x)$

Otven

Command $\boldsymbol{Y}$

RB : VN $(x) \rightarrow$ ITERATE $(x, x)$

R6 deleted

Rewrlte ruloe: Re R3 for left part

RT doloted

Rowrlte ruloo: RB RA for left part

RB RE for right pert 


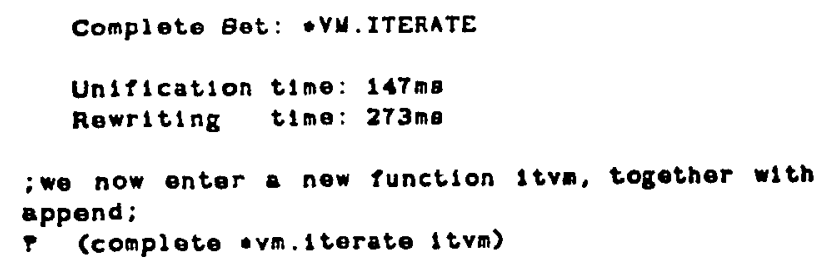

Given get of equationo: ITVM

$\operatorname{ITVM}(N U L L, x, y)=y$

$\operatorname{ITVM}(\operatorname{CONB}(x, y), z, u)=\operatorname{ITVM}(y, z, \operatorname{CON} \theta(x, u))$

APPEND (NULL, $x$ ) $=x$

$\operatorname{APPEND}(\operatorname{CONB}(x, y), x)=\operatorname{CONB}(x, A P P E N D(y, z))$

RT : ITVM(NULL, $x, y) \rightarrow y$

Given

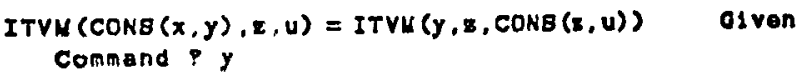

R10 : APPEND $(\operatorname{CONB}(x, y), z) \rightarrow \operatorname{CONB}(x, \operatorname{APPEND}(y, z))$

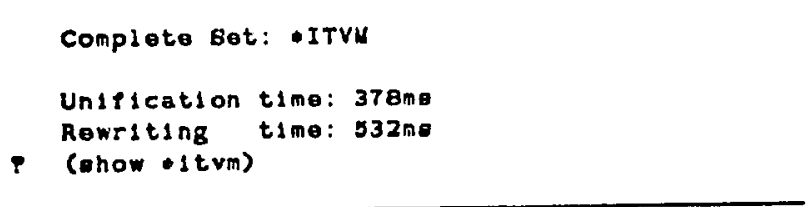

\section{- ITVK}

DCONE $(x$, NULL $) \rightarrow$ NULL $\operatorname{CONB}(x, \operatorname{CONB}(y, z)) \rightarrow \operatorname{CONB}(\operatorname{CONB}(x, y), \operatorname{DCONB}(x, z))$ ITERATE (NULL, $x) \rightarrow$ NULL ITERATE $(\operatorname{CONB}(x, y), z) \rightarrow \operatorname{CONB}(z, \operatorname{ITERATE}(y, z))$ $\operatorname{DCONB}(x, \operatorname{ITERATE}(y, z)) \rightarrow \operatorname{ITERATE}(y, \operatorname{CONB}(x, z))$ $\operatorname{VM}(x) \rightarrow$ ITERATE $(x, x)$ ITVN (NULL, $x, y) \rightarrow y$ $\operatorname{ITVN}(\operatorname{CONB}(x, y), z, u) \rightarrow \operatorname{ITVN}(y, z, \operatorname{CONB}(z, u))$ APPEND (NULL, $x$ ) $\rightarrow x$ $\operatorname{APPEND}(\operatorname{CON} B(x, y), z) \rightarrow \operatorname{CONB}(x, \operatorname{APPEND}(y, z))$

; we now expreas ltvm in terme of 1terate; p (prove itva.1terate)

olven oet of equationo: ITVu.ITERATE $\operatorname{ITVM}(x, y, z)=A$ PPEND $(\operatorname{ITERATE}(x, y), z)$

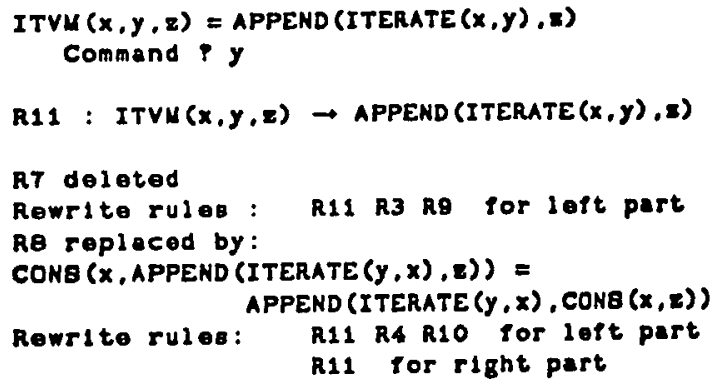

Complete Bot: DITYM.ITERATE

Unification time: $324 m \theta$

Rewriting tine: b81mo

;and finally, we whow that wo may compute $v$ iteretively, alnce: $\quad v m(x)=1$ tva $(x, x, n u l l)$. This proof le done by mere elmpliflcetion, once wo prove the lemma:

P (prove vm.1tvm) appond $(x, n \cup L 1)=x$ :

osven eot of equatione: VN.ITVu

$\operatorname{APPEND}(x$, NULL $)=x$

$V M(x)=\operatorname{ITVM}(x, x, N U L L)$

R11: APPEND $(x, N U L L) \rightarrow x$

OIven

Complete Bet: •VN. ITVM

Uniflcation time: $151 \mathrm{mB}$

Rewrlting time: 205mo

P (ahow vim.1tvm)

$\bullet$ VM . ITVI

$\operatorname{DCONB}(x, N U L L) \rightarrow$ MULL

$\operatorname{DCONB}(x, \operatorname{CONB}(y, z)) \rightarrow \operatorname{CONB}(\operatorname{CoN} 8(x, y), \operatorname{DCONB}(x, z))$

ITERATE (NULL, $x) \rightarrow$ NULL

ITERATE $(\operatorname{CONB}(x, y), z) \rightarrow \operatorname{CONB}(z, \operatorname{ITERATE}(y, z))$

$\operatorname{DCONB}(x, \operatorname{ITERATE}(y, z)) \rightarrow \operatorname{ITERATE}(y, \operatorname{CONB}(x, z))$

$\operatorname{VH}(x) \rightarrow$ ITERATE $(x, x)$

APPEND $(N U L L, x) \rightarrow x$

APPEND $(\operatorname{CONB}(x, y), z) \rightarrow \operatorname{CONB}(x, \operatorname{APPEND}(y, z))$

$\operatorname{ITVM}(x, y, z) \rightarrow$ APPEND (ITERATE $(x, y), z)$

APPEND (ITEAATE $(x, y), \operatorname{CONB}(y, z)) \rightarrow$

APPEND $(x, N U L L) \rightarrow x$
: We thank Patrick Greueray for euggeding the example above.

Let ue now glve an exanple of dieproot:

? (ohow rev) 


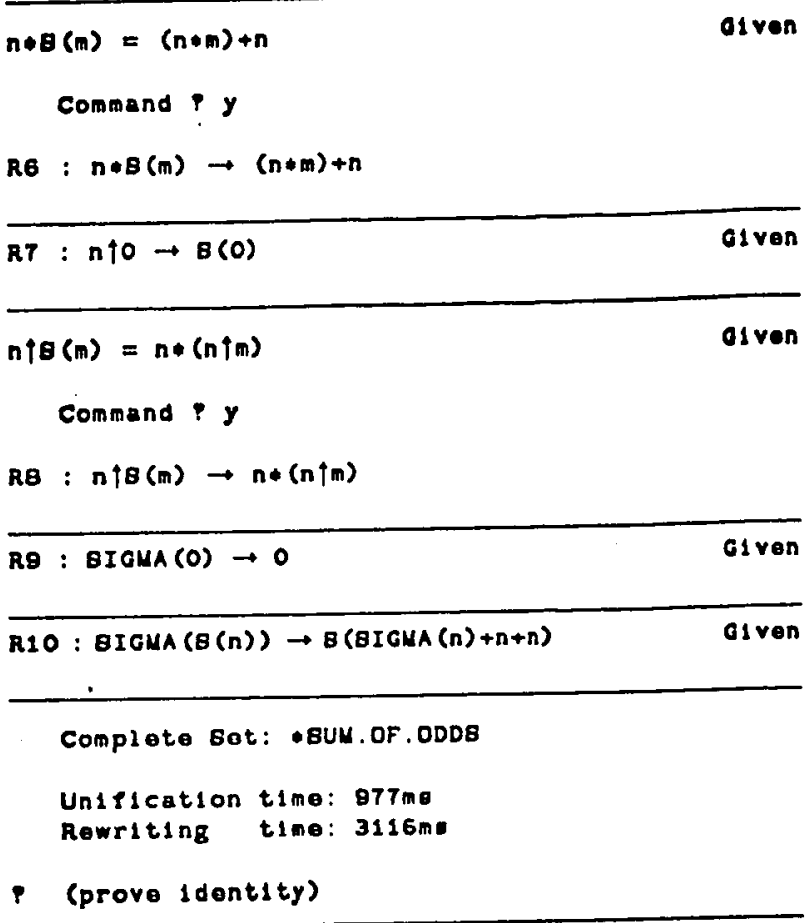

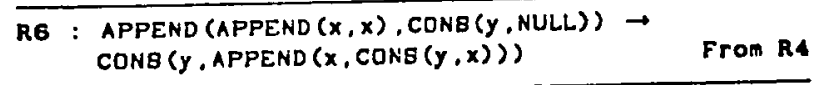

NULL $=\operatorname{CONB}(x$, NULL $)$ Fron $R 6$ and $R 1$

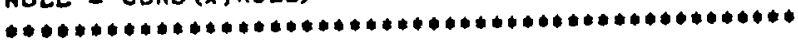

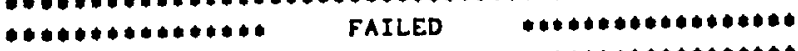

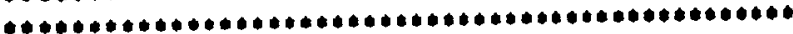

Wo now give examples of the method with commutative-anooclative operatore;

P (Initialization)

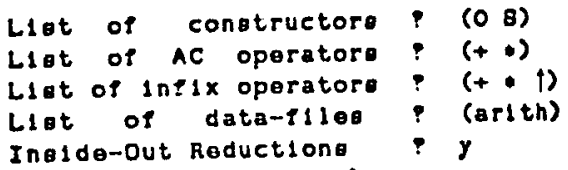

- (complete oum.of.odde)

olven eet of equatione: BUn.OF.ODDe

$1=B(0)$

$z=8(1)$

$n+0=n$

$n+B(m)=8(n+m)$

$n+0=0$

$n * 8(m)=(n * m)+n$

$n+0=1$

$n \nmid B(m)=n \cdot(n \nmid m)$

GIGKA $(0)=0$

$\operatorname{BIGNA}(B(n))=\operatorname{BIGMA}(n)+((2 * n)+1)$

\begin{tabular}{lr}
\hline$R 1: 1 \rightarrow B(0)$ & Given \\
\hline$R 2: 2 \rightarrow B(B(0))$ & Given \\
\hline$R 3: n+0 \rightarrow n$ & Given \\
\hline$R 4: n+8(m) \rightarrow 8(n+m)$ & Oiven \\
\hline
\end{tabular}

Given eot of equatione: IDENTITY

$\operatorname{\theta IanA}(n)=n \uparrow 2$

$\operatorname{\theta IGMA}(n)=n * n$

osvon

Command $y$

R11: $\operatorname{\theta IGMA}(n) \rightarrow \operatorname{nen}$

R日 deloted

Rowplte rulee : RIL RS for left part

R10 deleted

Rewrite ruieo: R11 R6 R4 R6 for left part

R11 for rlght part

Complete Bot: a IDENTITY
Unificetion time: some
Rewriting time: T42me
(ehow identity)

\footnotetext{
- IDENTITY

$1 \rightarrow 8(0)$

$2 \rightarrow B(B(0))$

$n+0 \rightarrow n$

$n+8(m) \rightarrow B(n+m)$

$n+0 \rightarrow 0$

$n * \theta(m) \rightarrow(n * m)+n$

$n \uparrow 0 \rightarrow 8(0)$

$n \uparrow B(m) \rightarrow n *(n \uparrow m)$

GIGMA $(n) \rightarrow n * n$
} 


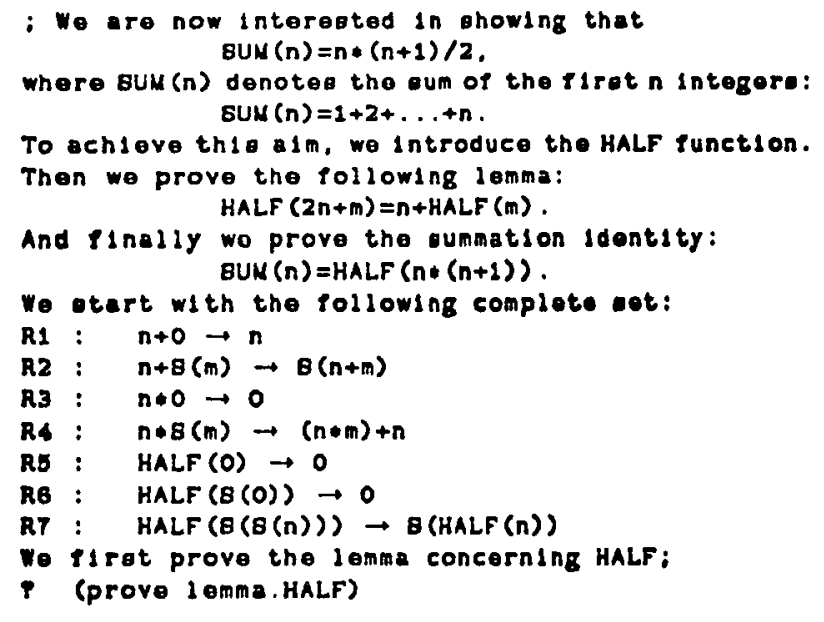

Given get of equatione: LEMMA.HALF

$\operatorname{HALF}(n+n+m)=n+H A L F(m)$

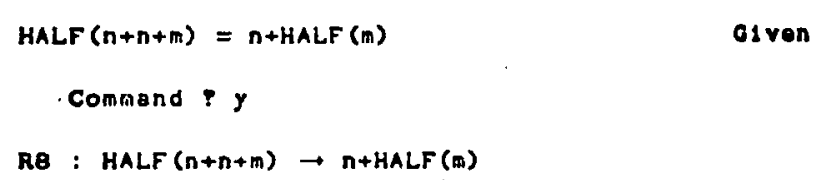

Complete Bet: + Lenha. half

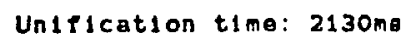

Qiven eet of equatione: DEF.BUM

$\operatorname{BUN}(0)=0$

$\operatorname{BUN}(B(n))=\operatorname{BUN}(n)+8(n)$

$R 12: \operatorname{BUM}(0) \rightarrow 0$

orvan

R13: BUM $(B(n)) \rightarrow B(B U N(n)+n)$

Given complete Bet: - DEF. BUM

Unlication time: 198me

Rewriting tine: stone

; we are now ready to prove the cummation Identity; (prove proor.oum)

Given eet of equatione: Proof.BUM

$\operatorname{BUN}(n)=\operatorname{HALF}(n * B(n))$
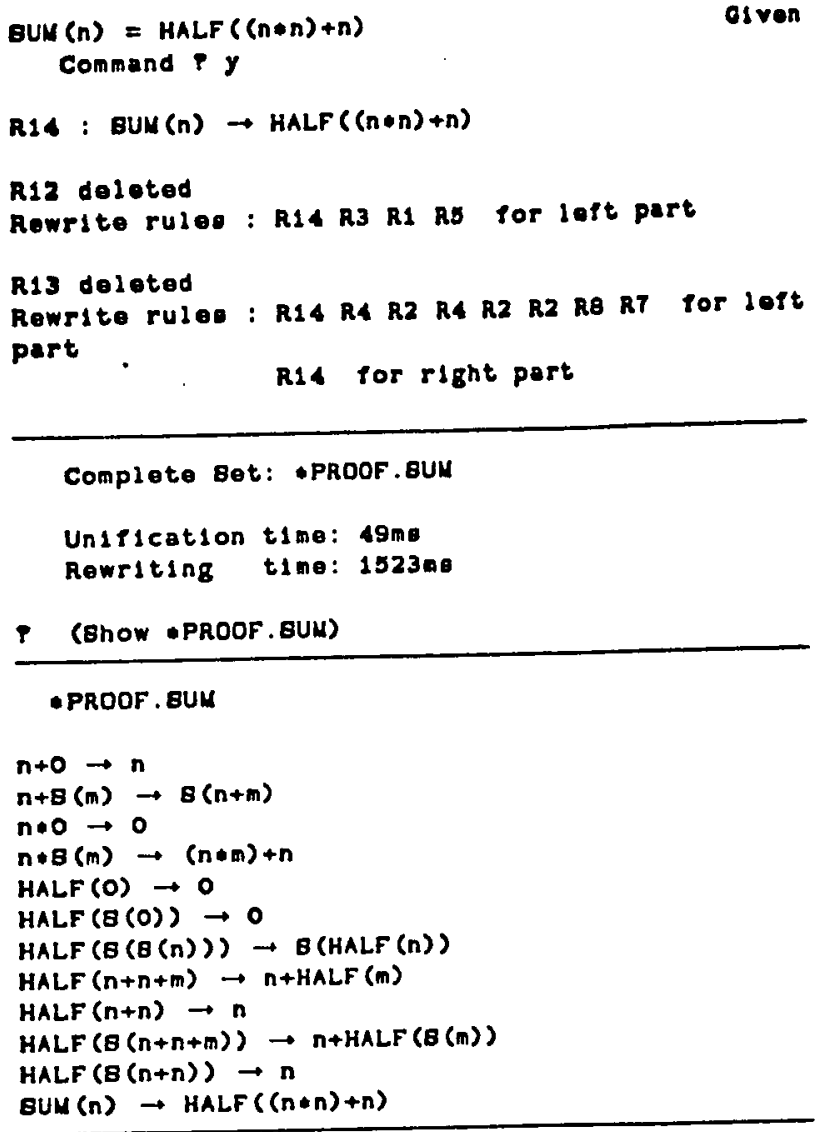

\section{Acknowlegments}

This research was carried out during a visit to the Computer Science Laboratory of SRI International. We bad numerous discussions on the topic of inductive proofs with Bob Boyer, Joe Goguen, Patrick Greussay, J Moore and Rob Shostak. We are very grateful to Jérôme Chailloux for transporting VLISP on the KL10 at SRI. We thank Stanford University for allowing us to typeset this paper in $\mathrm{TEX}_{\mathrm{E}}$ on the SAIl computer. 


\section{References}

1. Aubin R., Mechanizing Structural Induction. Ph.D. thesis, U. of Edinburgh, Edinburgh 1976.

2. Boyer R. and Moore J, Proving Theorems About LISP Functions. JACM 22 (1975), 129-144.

3. Boyer R. and Moore J, A Computational Logic. Academic Press, 1979.

4. Eurstall R.M., Proving Properties of Programs by Structural Induction. Computer J. 12 (1969), 4148.

5. Dershowitz N., Orderings for Term-rewriting Systems. Proc. 20th Symposium on Foundations of Computer Science (1979), 123-131. To appear, Theoretical Computer Science.

6. Goguen J.A., How to Prove Algebraic Inductive Hypotheses Without Induction, With Applications to the Correctness of Data Type Implementation. Proceedings of the Fifth Conference on Automated Deduction, Les Arcs, July 1980.

7. Goguen J.A., Thatcher J.W., Wagner E.G. and Wright J.B., Initial Algebras Semantics and Continuous Algebras. JACM 24 (1977), 68-95.

8. Goguen J.A., Thatcher J.W. and Wagner E.G., An Initial Algebra Approach to the Specification, Correctness, and Implementation of Abstract Data Types. "Current Trends in Programming Methodology", Vol 4, Ed. Yeh R., Prentice-Hall (1978), 80-149.

8. Huet G., Confluent Reductions: Abstract Properties and Applications to Term Rewriting Systems. 18th IEEE Sym posium on Foundations of Computer Science (1977), 30-45. To appear, JACM, October 1980 .

10. Huet G., A Complete Proof of Correctness of the Knuth-Bendix Completion Algorithm. Unpublished manuscript, March 1980. Rapport de Recherche INRIA to appear.

11. Huet G. and Oppen D., Equations and Rewrite Rules: a Survey. "Formal Languages: Perspectives and Open Problems". Ed. Book R., Academic Press, 1980. Also Technical Report CSL-111, SRI International, January 1980.

12. Kamin S. and Lévy J.J., Private communication. February 1980.

13. Knuth D. and Bendix P., Simple Word Problems in Universal Algebras. "Computational Problems in Abstract Algebra”. Ed. Leech J., Pergamon Press, 1970, 263-297.
14. Lankford D.S. and Ballantyne A.M., Decision Procedures for Simple Equational Theories With Commutative-Associative Axioms: Complete Sets of Commutative-Associative Reductions. Report ATP 39, Departments of Mathematics and Computer Sciences, U. of Texas at Austin, Aug. 1977.

15. Musser D. L., On Proving Inductive Properties of Abstract Data Types. Proceedings of the Seventh Annual ACM Sym posium on Principles of Programming Languages, Las Vegas, Jan. 1980, 154-162.

16. Peterson G.E. and Stickel M.E., Complete Sets of Reductions for Equational Theories With Complete Unification AJgorithms. Tech. Report, Dept. of Computer Science, U. of Arizona, Tucson, Sept. 1977.

17. Plaisted D., A Recursively Defined Ordering for Proving Termination of Term Rewriting Systems. Dept. of Computer Science Report 78-943, U. of Illinois at Urbana-Champaign, Sept. 1978. 
